\title{
miRNA profiling of B-cell subsets: specific miRNA profile for germinal center $B$ cells with variation between centroblasts and centrocytes
}

\author{
Lu Ping Tan ${ }^{1}$, Miao Wang ${ }^{1}$, Jan-Lukas Robertus ${ }^{1}$, Rikst Nynke Schakel ${ }^{1}$, Johan H Gibcus ${ }^{1}$, Arjan Diepstra ${ }^{1}$, \\ Geert Harms ${ }^{1}$, Suat-Cheng Peh ${ }^{2}$, Rogier M Reijmers ${ }^{3}$, Steven T Pals ${ }^{3}$, Bart-Jan Kroesen ${ }^{4}$, Philip M Kluin ${ }^{1}$, \\ Sibrand Poppema ${ }^{1}$ and Anke van den Berg ${ }^{1}$
}

MicroRNAs (miRNAs) are an important class of small RNAs that regulate gene expression at the post-transcriptional level. It has become evident that miRNAs are involved in hematopoiesis, and that deregulation of miRNAs may give rise to hematopoietic malignancies. The aim of our study was to establish miRNA profiles of naïve, germinal center (GC) and memory B cells, and validate their expression patterns in normal lymphoid tissues. Quantitative (q) RT-PCR profiling revealed that several miRNAs were elevated in GC B cells, including miR-17-5p, miR-106a and miR-181b. One of the most abundant miRNAs in all three B-cell subsets analyzed was miR-150, with a more than 10-fold lower level in GC B cell as compared with the other two subsets. miRNA in situ hybridization (ISH) in tonsil tissue sections confirmed the findings from the profiling work. Interestingly, gradual decrease of miR-17-5p, miR-106a and miR-181b staining intensity from the dark to the light zone was observed in GC. A strong cytoplasmic staining of miR-150 was observed in a minority of the centroblasts in the dark zone of the GC. Inverse staining pattern of miR-150 against c-Myb and Survivin was observed in tonsil tissue sections, suggesting possible targeting of these genes by miR-150. In line with this, the experimental induction of miR-150 lead to reduced c-Myb, Survivin and Foxp1 expression levels in the Burkitt's lymphoma cell line, DG75. In conclusion, miRNA profiles of naïve, GC and memory B cells were established and validated by miRNA ISH. Within the GC cells, a marked difference was observed between the light and the dark zone.

Laboratory Investigation (2009) 89, 708-716; doi:10.1038/labinvest.2009.26; published online 6 April 2009

KEYWORDS: germinal center; germinal center B cells; miRNA; miRNA in situ hybridization

MicroRNAs (miRNAs) are a class of small RNAs of 19-23 nucleotides that were discovered less than two decades ago. ${ }^{1,2}$ When incorporated into Argonaute proteins to form the RNAinduced silencing complex, miRNAs can negatively regulate genes at the post-transcriptional level by either triggering translational repression or direct cleavage of mRNAs. ${ }^{3}$ miRNA expression can be highly tissue-specific ${ }^{4}$ and is known to regulate many cellular processes, including proliferation, apoptosis, cell cycle, differentiation and hematopoiesis. ${ }^{5-8}$

The distinct and dynamic expression pattern of miRNAs during hematopoiesis reflects the importance of this class of small RNAs in determining the various states of differentiation of hematopoietic cells. ${ }^{9-12}$ Alteration in the expression level of merely one miRNA in hematopoietic stem cells can skew the differentiation process toward a specific hematopoietic cell type. ${ }^{13}$ For this reason, it was assumed that aberrant expression of miRNAs could be involved in the development of hematopoietic malignancies. Indeed, the specific involvement of a number of miRNAs not only in hematopoietic development, but also in lymphomagenesis, has been the subject of a growing number of studies in the past few years.

c-Myb, a transcription factor known to play a key role in B cell maturation, has been shown to be a direct target of miR$150 .{ }^{14}$ Ectopic expression of miR-150 in hematopoietic stem

\footnotetext{
'Department of Pathology and Laboratory Medicine, University Medical Center Groningen, University of Groningen, Groningen, The Netherlands; ${ }^{2}$ Department of Pathology, University Malaya Medical Center, University of Malaya, Kuala Lumpur, Malaysia; ${ }^{3}$ Department of Pathology, Academic Medical Center, University of Amsterdam, Amsterdam, The Netherlands and ${ }^{4}$ Department of Medical Biology, University Medical Center Groningen, University of Groningen, Groningen, The Netherlands

Correspondence: Dr A van den Berg, PhD, Department of Pathology and Laboratory Medicine, University of Groningen, University Medical Center Groningen, PO Box 30.001, Hanzeplein 1, Groningen 9700 RB, The Netherlands.

E-mail: a.van.den.berg@path.umcg.nl

Received 28 December 2008; revised 11 February 2009; accepted 13 February 2009
} 
cells leads to the blockage of the transition of pre- $\mathrm{B}$ to pro- $\mathrm{B}$ stage. ${ }^{15}$ The PU.1 transcription factor, essential in hematopoietic lineage development, and activation-induced cytidine deaminase (AID), a protein that is important in class switch recombination of the immunoglobulin locus, have been shown to be the targets of miR-155. ${ }^{16,17}$ As a consequence of miR-155 downregulation, inefficient germinal center (GC) response causing production of less $\mathrm{IgG}_{1}$-positive cells was observed. Li et $a l^{18}$ showed that miR-181a can fine-tune T-cell sensitivity during the maturation process by directly targeting SHP-2, PTPN22, DUSP5 and DUSP6, four components of the T-cell receptor signaling pathway. In parallel with the discoveries of important hematopoietic miRNAs, it has become evident that miRNAs are involved in the pathogenesis of leukemia and lymphoma. For example, miR-21, miR-92, miR-150, miR-155 and miR-222 are reported to be deregulated in chronic lymphocytic leukemia (CLL). ${ }^{19,20}$ Overexpression of miR-155 as well as the miR-17-92 cluster has been documented for several B-cell lymphomas, including diffuse large B-cell lymphoma and Hodgkin lymphoma. ${ }^{21-23}$ In a transgenic mouse model, ectopic expression of miR-155 leads to lymphoblastic leukemia/high-grade lymphoma. ${ }^{24}$

Recently, several studies have been carried out to establish miRNA profiles of hematopoietic cells, ${ }^{25,26}$ but none of them are confirmed by in situ hybridization (ISH). The aim of our study was to characterize the miRNA expression profile of normal B-cell subsets, which includes naïve, GC and memory B cells. As gene expression analysis identified only limited differences between centroblasts and centrocytes, ${ }^{27,28}$ both cell types were grouped as GC B cells in our study. By comparing these profiles, GC B-cell-specific miRNA expression patterns were defined. Findings from the profiling work were validated with miRNA ISH technique and expressions of three miR-150 targets was examined.

\section{MATERIALS AND METHODS Tissue Sections}

Tissue samples of hyperplastic tonsils removed from children with chronic tonsillitis were obtained from the Department of Ear Nose Throat, University Medicine Center Groningen and Academic Medical Center. All the protocols for obtaining and studying human tissues and cells were approved by the institution's review board for human subject research.

\section{FACS-Sorted Normal B-Cell Subsets}

Normal B-cell subsets were sorted by FACS from three different tonsil samples, essentially as described earlier. ${ }^{29}$ Briefly, mononuclear cells were isolated by Ficoll-Isopaque density gradient centrifugation. The collected cell suspension was stained with FITC-conjugated anti-human IgD, PE-conjugated anti-human CD19 (both from Dako, Glostrup, Denmark) and allophycocyanin-conjugated anti-human CD38 (BD Pharmingen, NJ, USA). Naïve B cells $\left(\mathrm{CD} 19^{+}\right.$, $\left.\mathrm{IgD}^{+}, \mathrm{CD}_{3} 8^{-}\right), \mathrm{GC} \mathrm{B}$ cells $\left(\mathrm{CD} 19^{+}, \mathrm{IgD}^{-}, \mathrm{CD} 38^{+}\right)$and memory B cells $\left(\mathrm{CD} 19^{+}, \mathrm{IgD}^{-}, \mathrm{CD} 38^{-}\right)$were isolated using a FACS aria (BD Biosciences, San Jose, USA). The cells were gated for single cells by forward and sideward scattering. After sorting, the B-cell subsets were lysed in RNA-Bee (Tel-Test Inc. Frindswood, TX, USA) and stored at $-80^{\circ} \mathrm{C}$, until further processing.

\section{qRT-PCR and Clustering Analysis}

The RNA was isolated from normal B-cell subsets with Nucleospin RNA 11 (Macherey-Nagel, Düren, Germany) as described earlier. ${ }^{29}$ B-cell subset RNA from three different tonsil samples were pooled together accordingly to rule out small differences related to genetic variation in individuals and differences related to histology. We used a commercially available miRNA quantitative RT-PCR (qRT-PCR) profiling kit (Applied Biosystems, Foster City, USA), which, at that time, contained 183 miRNAs. We did not specifically select the miRNAs. qRT-PCR profiling for 183 mature miRNAs were carried out according to manufacturer's protocols. Unsupervised clustering analysis was carried out using Genesis. ${ }^{30}$ A heatmap was generated by mean normalization of experiments and genes, followed by average linkage clustering for all miRNAs that showed a cycle threshold $(\mathrm{Ct})$ value of $<35$ in at least one of the three B-cell subsets. As the mean and median values for the three B-cell subsets were very similar (Ct value of 31.3-31.9), the mean of all miRNAs assessed was used for normalization $\left(\Delta \mathrm{Ct}=\mathrm{Ct}_{\text {miRNA }}-\mathrm{Ct}_{\text {mean }}\right)$. The relative expression levels were determined using the formula $2^{-\Delta C}$. Selection of GC B-cell-specific miRNAs was carried out by the following criteria: (1) miRNAs with $2^{-\Delta \mathrm{Ct}}$ $>2$ in at least one of the subsets, that is, miRNAs with a relatively high expression level and (2) miRNA showing at least 4.5 -fold difference among the subsets.

\section{miRNA In Situ Hybridization}

The miRNA ISH was carried out as reported earlier. ${ }^{31}$ Briefly, 11 digoxigenin (DIG)-labeled locked nucleic acid probes antisense to miR-15b, miR-17-5p, miR-21, miR-25, miR-29a, miR-93, miR-106a, miR-146a, miR-122, miR-150 and miR181b (Exiqon, Vedbaek, Denmark) were used for overnight hybridization on tissue sections at $55^{\circ} \mathrm{C}$. Detection was accomplished with anti-DIG alkaline phosphate Fab fragment followed by nitro blue tetrazolium chloride/5-bromo-4chloro-3-indolyl phosphate (NBT/BCIP) color development (Roche, Switzerland). miR-122 was chosen as a negative control for staining in tonsil sections, because it has been reported that the expression of miR-122 is detected only in liver. $^{32}$

\section{Immunohistochemistry}

Slides were deparaffinized and endogenous peroxidase was blocked by incubation with $3 \% \mathrm{H}_{2} \mathrm{O}_{2}$ for $10 \mathrm{~min}$. Antigen retrieval was carried out according to various protocols of the manufacturers. Immunostaining was carried out using antibodies against Survivin (Cell Signaling Technology, Boston, 
MA, USA), Foxp1 and c-Myb (Abcam, Cambridge, UK) at a dilution of 1:50-1:400. Signals were amplified by incubation with the appropriate horseradish peroxidase-conjugated antibodies for $60 \mathrm{~min}$ and the reactivity was visualized using diaminobenzidine.

\section{Transfection, Western Blotting and DiOC6 Staining}

The Epstein-Barr virus negative Burkitt's lymphoma (BL) cell line, DG75, ${ }^{33}$ was cultured in RPMI 1640 supplemented with ultraglutamine, $100 \mathrm{U} / \mathrm{ml}$ penicillin/streptomycin, and $10 \%$ fetal calf serum (Cambrex Biosciences, Walkersville, USA). Transfection of DG75 with synthetic miR-150 mature duplex (Ambion, Austin, USA) was carried out using the A-23 program of the Amaxa nucleofector I device (Amaxa, Gaithersburg, USA) with nucleofection solution V. At $24 \mathrm{~h}$ post-transfection, half of the cells were lysed, separated in $10 \%$ SDS-polyacrylamide gel and immunoblotted for $\mathrm{c}-\mathrm{Myb}$, Foxp1 and Survivin with the same antibodies as used for IHC. At $48 \mathrm{~h}$ post-transfection, the other half of the cells were incubated with $25 \mu \mathrm{g} / \mathrm{ml}$ DiOC6 at $37^{\circ} \mathrm{C}$ for $30 \mathrm{~min}$, washed and analyzed by FACS. Cells showing high DiOC6 staining were gated as live cells, whereas cells with low DiOC6 staining were regarded as apoptotic/death cells.

\section{RESULTS}

miRNA Profiling For Normal B-Cell Subsets From Tonsils Out of 183 miRNAs assessed, 86 miRNAs were expressed $(\mathrm{Ct}<35)$ in at least one of the three normal B-cell subsets. A heatmap of these 86 miRNAs is given in Figure 1a. To identify miRNAs that are important throughout the GC response, we focused on the miRNAs that showed at least 4.5-fold difference between GC and non-GC B cells, resulting in $16 \mathrm{miR}$ NAs (Figure 1b). A total of 8 of these 16 miRNAs, namely miR-15b, miR-93, miR-181b, miR-25, miR-17-5p, miR-106a, miR-28 and miR-130b showed increased expression levels in GC B cells, whereas miR-150, miR-29a, miR-320 and miR-223 showed a lower expression level in GC B cells. The expression levels of miR-145 and miR-146 increased during the GC transit, whereas the expression levels of miR-331 and let-7a decreased during the GC transit (Figure 1b, Table 1).

\section{miRNA ISH In Tonsils}

In view of the differential miRNA expression among the normal B-cell subsets and our main interest in GC B cells, nine miRNAs, which were most abundant and differentially expressed in GC B cells, (the first 9 miRNA listed from the left, Figure 1b) were chosen for miRNA ISH. As the differences in miR-21 expression level among the three B-cell subsets were minimal ( $\sim$ twofold differences, Table 1$)$, this miRNA was chosen as a control for ISH, in which we expect to see no variation in staining intensity within the GC structures. Meanwhile, the liver-specific miR-122 probe was used as the negative control. Out of the 11 miRNAs chosen for $I S H$, staining for four miRNAs (miR-15b, miR-93, miR25 and miR-29a) failed in all the cases for unknown reasons.
This failure was not related to the abundance of the miRNAs in GC B cells, as determined by qRT-PCR profiling.

As a negative control, ISH with miR-122, a liver-specific probe, showed negative staining in the tonsil tissue section (Figure 2). miR-21 showed no variation in the staining intensities of GC structures and the mantle zone in tonsil tissue sections as expected. According to morphology and distribution pattern in the tonsil, cells with the strongest miR-21 staining were likely to be interfollicular T cells (Figure 2). The proteinase $\mathrm{K}$ step in the $\mathrm{ISH}$ procedure precludes double staining of miR-21 and CD3, therefore, we can only suggest that the cells with the strongest miR-21 staining in tonsils represent interfollicular $\mathrm{T}$ cells based on their morphology and distribution pattern in the tonsil.

The miRNA ISH results showed weak cytoplasmic staining of miR-150 in GC as compared with the mantle zone, whereas miR-17-5p, miR-106a and miR-181b stained stronger in GC structures (Figure 2). This indicates a higher expression of miR-17-5p, miR-106a and miR-181b and a lower expression of miR-150 in GC B cells as compared with naïve and memory B cells located in the mantle zone (Figure 2). These results were consistent with our miRNA profiling results. For the staining of miR-146a, our miRNA ISH depicted a stronger staining in the GC (Figure 2), mainly contributed by a stronger staining in the centroblasts in the dark zone of the GC, as compared with a weaker signal in the mantle zone (Figure 3). Profiling showed a low expression in naïve cells as compared with GC and memory B cells, which showed an almost similar expression level. As naïve B cells (low miR-146a levels) represent a major population in the mantle zone as compared with the memory cells (high miR-146a levels), this might explain the stronger staining observed in the GC B cell dark zone (Figure 2).

Interestingly, variation in staining intensity was observed within the GC structures for several miRNAs. Gradual decrease of miR-17-5p, miR-106a and miR-181b staining was observed from the dark to the light zone in the GC (Figure 2). miR-150 showed the strongest staining in part of the centroblasts only in the dark zone, whereas the light zone, containing the centrocytes, stained even weaker than the naïve and the memory B cells in the mantle zone (Figure 3 ).

\section{miR-150 Expression Levels and Its Possible Role In B-Cell Homeostasis}

In view of the dynamic expression patterns of miR-150 throughout the GC reaction and its absence in proliferation centers of CLL cases, ${ }^{20}$ we investigated the possible role of miR-150 in regulation of B-cell homeostasis. One proven target for miR-150, c-Myb, ${ }^{14}$ and two putative miR-150 targets, Foxp1 (both PICTAR and TargetScan 4.2 predictions) and Survivin (not predicted by both PICTAR and TargetScan 4.2 programs, but contained miR-150 6mer seed complimentary site at its $3^{\prime}$ UTR), were examined by immunohistochemistry staining. The $\mathrm{c}-\mathrm{Myb}$ and Survivin staining patterns were inversely correlated with miR-150 ISH 
a
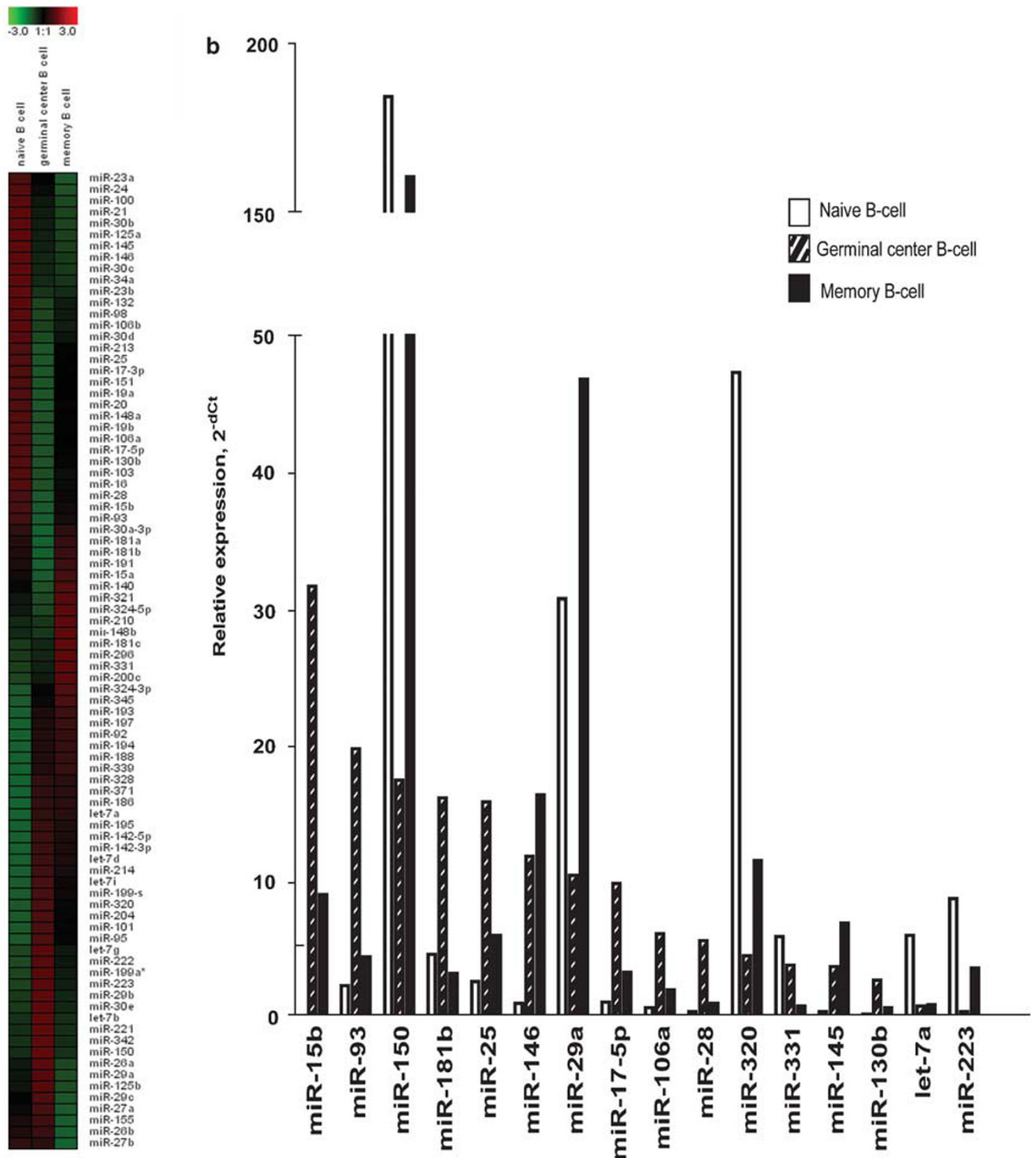

Figure 1 miRNA profiling of naïve, germinal center (GC) and memory B cells. (a) Heatmap of 86 miRNAs, which were expressed $(C t<35)$ in at least one of the normal B-cell subsets and showed differential expression among the subsets. Green color represents lower $\mathrm{Ct}$ value, that is, upregulation, whereas red color represents higher $\mathrm{Ct}$ value, that is, downregulation. (b) 16 miRNAs showed at least 4.5 -fold difference when compared with GC B cells. The first nine miRNAs and miR-21 (control with not more than twofold changes during the GC transit, not shown in graph) were selected for ISH.

staining patterns in tonsils (Figure 4a), supporting the targeting of these genes by miR-150. The staining pattern of Foxp1 did not show a clear inverse pattern with miR-150 ISH (Figure 4a). To confirm targeting by miR-150, the BL cell line, DG75, which has a GC B-cell origin and low miR-150 expression level, was transfected with synthetic miR-150 mature duplex for induction of the miRNA (confirmed by
qRT-PCR, data not shown). Western blot analysis of cell lysates showed repression of c-Myb, Survivin as well as Foxp1 in the cells transfected with miR-150 (Figure 4b). These results suggest that the expression of miR-150 either directly or indirectly regulates the expression of c-Myb, Survivin and Foxp1, three genes that are important during $\mathrm{B}$ cell development. ${ }^{34,35}$ In addition, DiOC6 analysis for DG75 cell 
Table 1 Germinal center-specific miRNAs

\begin{tabular}{|c|c|c|c|c|c|}
\hline & \multicolumn{3}{|c|}{ Relative expression, $2^{-\Delta \mathrm{Ct}}$} & \multicolumn{2}{|c|}{ Fold change } \\
\hline & Naïve B cell & GC B cell & Memory B cell & $\mathrm{GC} / \mathrm{N}$ & GC/M \\
\hline \multicolumn{6}{|l|}{ Highest in GC } \\
\hline miR-15b & 4.8 & 31.5 & 8.9 & 6.5 & 3.5 \\
\hline miR-93 & 2.2 & 19.6 & 4.3 & 8.7 & 4.5 \\
\hline miR-181b & 4.5 & 16.0 & 3.1 & 3.5 & 5.2 \\
\hline $\operatorname{miR}-25$ & 2.5 & 15.7 & 5.9 & 6.2 & 2.7 \\
\hline miR-17-5p & 1.0 & 9.7 & 3.2 & 9.8 & 3.0 \\
\hline miR-106a & 0.6 & 6.0 & 1.9 & 10.5 & 3.1 \\
\hline miR-28 & 0.3 & 5.5 & 0.9 & 19.9 & 5.9 \\
\hline miR-130b & 0.1 & 2.6 & 0.6 & 21.2 & 4.0 \\
\hline
\end{tabular}

Lowest in GC

$\begin{array}{rrrrrr}\text { miR-150 } & 184.1 & 17.3 & 160.5 & 0.1 & 0.1 \\ \text { miR-29a } & 30.6 & 10.3 & 46.7 & 0.3 & 0.2 \\ \text { miR-320 } & 47.2 & 4.4 & 11.4 & 0.1 & 0.4 \\ \text { miR-223 } & 8.6 & 0.3 & 3.5 & 0.0 & 0.1\end{array}$

Change during GC transit

Increase

$\begin{array}{rrrrrr}\text { miR-145 } & 0.3 & 3.6 & 6.8 & 13.4 & 0.5 \\ \text { miR-146 } & 0.9 & 11.7 & 16.2 & 12.6 & 0.7\end{array}$

Decrease

$\begin{array}{llllll}\text { miR-331 } & 5.8 & 3.7 & 0.7 & 0.6 & 5.5 \\ \text { let-7a } & 5.9 & 0.7 & 0.8 & 0.1 & 0.9\end{array}$

Consistent

miR-21

7.5

15.1

18.2

2.0

0.8

GC, germinal center. N, naive B cell. M, memory B cell. lines $48 \mathrm{~h}$ post-transfection with miR-150 showed that there was an increase in the percentage of cells with low DiOC6 staining (Figure 4c). Although the increase was not statistically significant, the trend indicates that the amount of apoptotic/death cells increased upon transfection with synthetic miR-150 mature duplex.

\section{DISCUSSION}

Results of miRNA ISH on tonsil sections confirmed differential expression of several miRNAs, as identified by the miRNA profiling work. More than 4.5 -fold changes in the expression levels of miR-17-5p, miR-106a, miR-146a, miR-150 and miR-181b were found in GC B cells as compared with naïve and memory B cells. A GC B-cell-specific expression pattern was confirmed by miRNA ISH staining. To our knowledge, this is the first report confirming distinct miRNA expression patterns in tonsils with ISH. Recently, Malumbres $e a^{26}$ did a similar profiling to identify miRNA expression changes during the GC response, but only centroblasts and not centrocytes were used. A total of 39 miRNAs were identified as the classifier to distinguish among $\mathrm{T}$ cells, centroblasts, naïve and memory B cells. Our study examined the GC B cells, which included centroblasts and centrocytes, looked for miRNAs with the highest fold change (>4.5-fold) in naïve or memory compared with GC B cells ( $\mathrm{T}$ cells not included) and resulted in a total of 16 miRNAs. Despite the differences in cell sorting and profiling technique, we observed a similar expression pattern for 28 out of their 39 classifier miRNAs (Supplementary Data 1). A total of 12 of the 16 miRNAs showing the most extreme changes in the expression levels during GC response in our study are included in their classifier miRNAs. We confirmed differences between GC and naïve/memory B cells for five miRNAs by ISH in tonsil tissue sections. We show that ISH is a powerful tool to study cell or compartment-specific miRNA expression, which can easily be missed upon analysis from sorted cells.
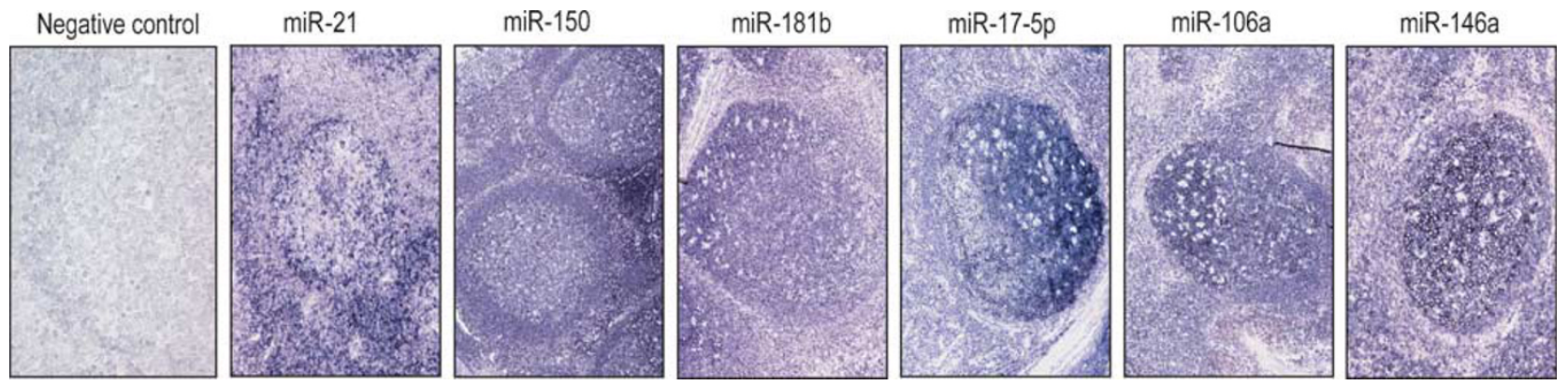

Figure 2 miRNA expression patterns in tonsils. Tonsil tissue section stained negative when miR-122, a liver-specific probe is used. Staining intensity of miR-21 does not vary between the GC and the mantle zone (MZ), cells with strong positive staining are likely to be interfollicular $T$ cells. miR-150 stains weaker in the GC as compared with the MZ. miR-181b, miR-17-5p and miR-106a stained stronger in the GC than in the MZ, with gradual decrease in the intensity observed from the dark zone to the light zone of the GC. Staining intensity of miR-146a appeared to be stronger in the GC than in the MZ. $\times 10$ magnification is shown for all tonsils. 

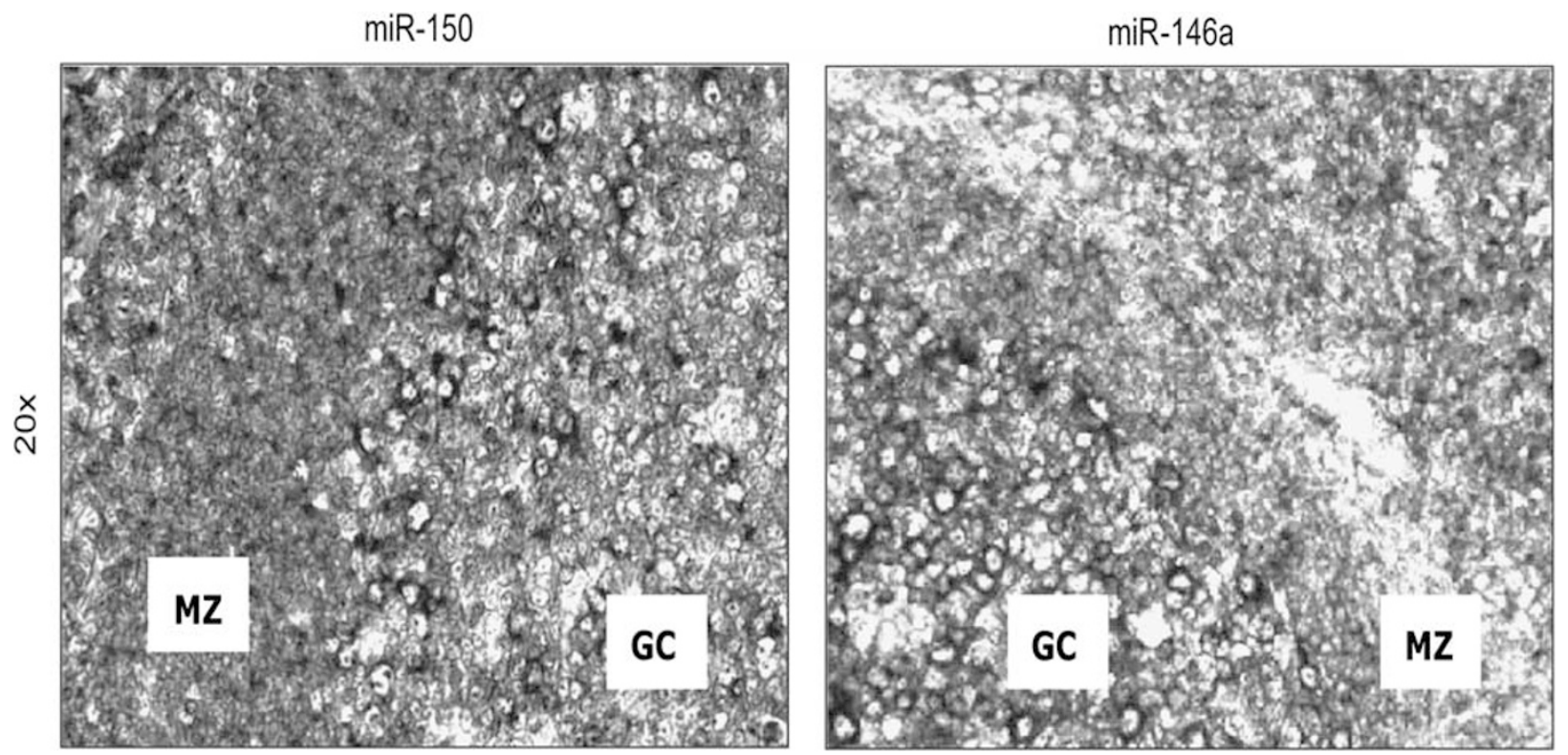

Figure 3 Close up pictures for miRNA staining patterns seen in germinal center (GC) structures of normal tonsils. Staining intensity of miR-150 was weaker in the GC as compared with the mantle zone (MZ), but some cells in the GC, likely centroblasts, have the strongest staining. Staining intensity of miR-146a appeared to be stronger in the GC, and most cells, which have the strongest staining in the GC, are likely to be centroblasts. The MZ with mixture of naïve and memory B cells stained weaker compared with the GC, presumably because of the domination of naïve B cells (with lower miR-146a expression) in the GC.

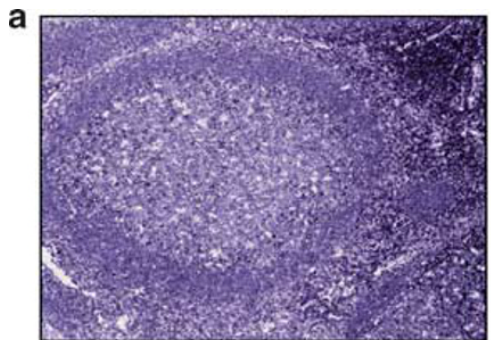

miR-150

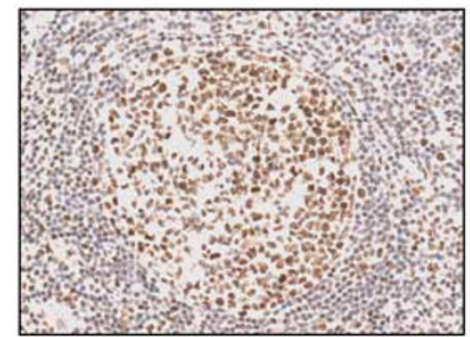

Survivn

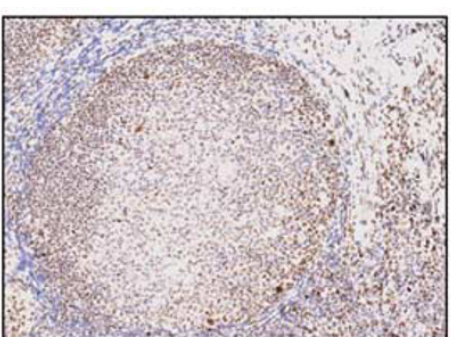

c-Myb

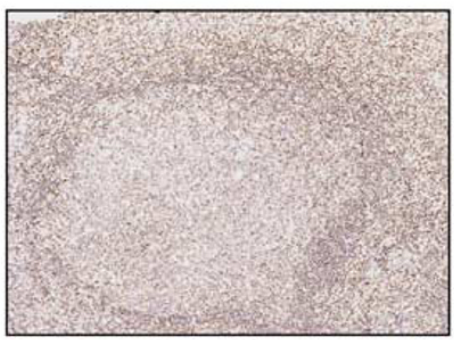

Foxp1
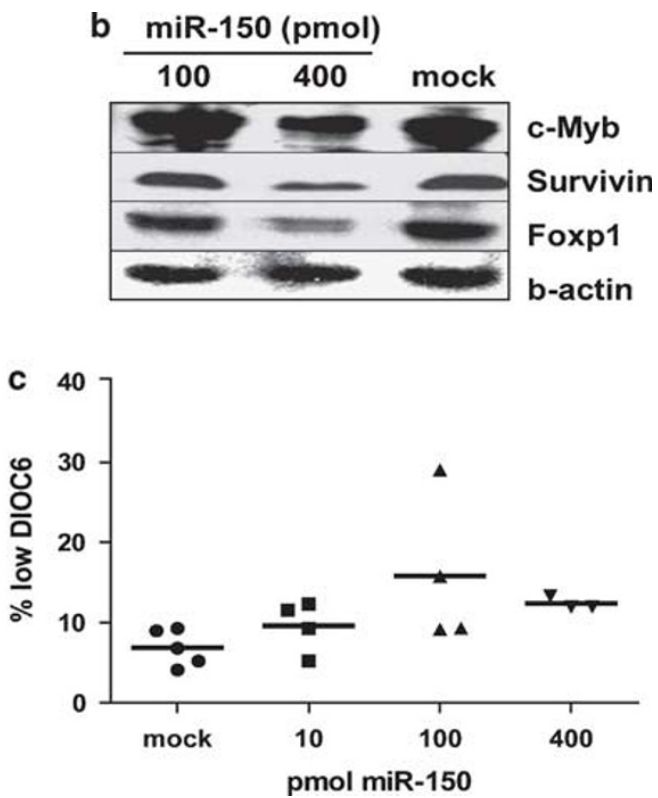

Figure 4 miRNA target genes validation. (a) Immunohistochemistry staining showing that the expression of c-Myb $(\times 10)$ and Survivin $(\times 20)$, but not Foxp1 ( $\times 10$ ), were inversely correlated to miR-150 in tonsils. (b) Downregulation of c-Myb, Survivin and Foxp1 upon miR-150 overexpression in DG75. (c) Although not statistically significant, an increase in the percentage of apoptotic/death cells (low DIOC6 staining) was observed when miR-150 was induced in DG75.

The miRNA ISH revealed a distinct staining pattern in the GC structures with decreasing miR17-5p, miR-106a and miR-181b levels from the dark zone to the light zone. This difference was most likely caused by difference in the expression levels between centroblasts and centrocytes. CD77 is generally accepted as a marker to discriminate between centroblasts and centrocytes. ${ }^{36,37}$ In earlier reported gene expression profiling studies of B cells during GC transit, 
Klein et $a l^{28}$ found thousands of differences among naïve, GC and memory B cells, but only 19 differentially expressed genes between $\mathrm{CD}^{-} 7^{-}$and $\mathrm{CD} 77^{+}$GC B cells. Consistent with these findings, Hogerkorp and Borrebaeck ${ }^{27}$ also found no differences in the gene expression profiles between CD77 ${ }^{-}$ and $\mathrm{CD}_{77^{+}}$GC B cells. Lack of differences in these studies raised the question whether CD77 can be used as a reliable marker to discriminate between centroblasts and centrocytes. In view of the dynamics of GC B cells to shift from the dark to the light zone, ${ }^{38}$ and because of the current limitations in isolating pure centroblasts and centrocytes, miRNA $I S H$ in normal tissue represents the best tool to study putative differences in miRNA expression levels in these two closely related B cell populations, as differences in ISH staining intensities were obviously seen in cells within the GC.

MiR-17-5p and miR-106a are both members of the same seed family, namely the miR-17 family, which also includes miR-17-5p, miR-20a, miR-20b, miR-93, miR-106a and miR106b. These miRNAs share the same seed sequence and most likely target the same genes, and hence exert similar effects. It has been shown that miRNAs from this miR-17 seed family negatively regulate the expression of cyclin-dependent kinase inhibitor, CDKN1A (also known as p21), allowing cells to overcome the G1 cell cycle checkpoint. ${ }^{39}$ As high expression levels of miR17-5p and miR-106a were observed specifically in centroblasts in the dark zone of GC, whereas CDKN1A has been reported to be 30 -fold downregulated in centroblasts, we suggest that these miRNAs are essential for centroblasts to progress from G1 to $S$ phase of the cell cycle, by downregulating CDKN1A. ${ }^{27,28}$

The expression level of miR-146a increases dramatically when the naive $\mathrm{B}$ cells enter the $\mathrm{GC}$ and remains high when they exit from the GC. It has been reported that proinflammatory cytokines and microbial components can induce the expression of miR-146a in the acute monocytic leukemia cell line, THP-1. ${ }^{40}$ Luciferase reporter assays showed that miR-146a is a nuclear factor- $\kappa \mathrm{B}$ (NF- $\kappa \mathrm{B}$ )dependent miRNA. ${ }^{40}$ By targeting IL-1 receptor-associated kinase and TNF receptor-associated factor 6, miR-146a causes a negative feedback for the Toll-like receptor signaling pathway. ${ }^{40}$ As the role of NF- $\kappa \mathrm{B}$ in B-cell homeostasis has been implicated in both immature $B$ cell and also in maintaining GC function, ${ }^{41}$ the strong miR-146a staining pattern that we observed in GC correlates with the functional role of NF- $\kappa \mathrm{B}$ in the GC. However, the exact function of miR-146a in this pathway and how it might affect B cell survival should be further investigated.

MiR-181b contains the same seed sequence as miR-181a. MiR-181a has been proven to play a role in hematopoietic differentiation, in favor of $\mathrm{B}$ cells ${ }^{13}$ and its expression is regulated during $\mathrm{T}$ cell maturation. ${ }^{18}$ Recently, it has been shown that AID is a direct target of miR-181b and overexpression of miR-181b in primary splenic B cells reduces the class switch recombination rate. ${ }^{42}$ We observed dynamic expression of both miR-181a and miR-181b during the GC transit, in which their expression levels peak during the GC stage and fall back to a level similar to that of naïve B cells upon exit from the GC. qRT-PCR profiling revealed a twofold and a fivefold enrichment in GC B cells for miR-181a and miR-181b, respectively. These results indicate that both miR-181a and miR-181b from the same miRNA seed family are crucial for $\mathrm{B}$ cell differentiation and fine-tuning of the $\mathrm{GC}$ response.

Our group demonstrated that miR-150 is highly expressed in the majority of CLL tumor cells, but not in the proliferation centers. ${ }^{20}$ CLL cells are considered to be of memory B-cell origin or derived from naïve B cells. ${ }^{43,44}$ The high miR150 expression in CLL is consistent with the high miR-150 expression levels that we found in naïve and memory B cells sorted from normal tonsil, and the lack in proliferation centers is consistent with the low levels in GC structures of tonsil. Recently, a key role has been established for miR-150 in B cell development. ${ }^{14,15}$ Overexpression of miR-150 blocks $\mathrm{B}$ cell maturation by inhibiting the transition of cells from pro-B to pre-B stage and also induces a slight, but significant, increase in apoptotic rate. ${ }^{15}$ Consistent with this finding, Xiao et al $^{14}$ showed that ectopic miR-150 expression in pro-B cells resulted in an increase in cell death. It was concluded that miR-150 exerts this effect by suppression of its target, $\mathrm{c}-\mathrm{Myb},{ }^{14}$ a transcription factor which plays an important role during $B$ cell development, maintenance of proliferation as well as cell cycle control of hematopoietic cells. ${ }^{35,45-47} \mathrm{c}-\mathrm{Myb}$ has already been proven to be important for transition of pro- $B$ to pre-B stage and also for maintenance of follicular $B$ cells, but interestingly, not for mantle zone B cells. ${ }^{35}$ This is in line with our findings, in which in the mantle zone, the expression of c-Myb indeed was weak, whereas the expression of miR-150 that negatively regulates $\mathrm{c}-\mathrm{Myb}$ was found to be relatively higher. Similarly, in tonsil sections, Survivin showed an inverse staining pattern with miR-150. For Foxp1, however, such an inverse staining pattern was not obvious, because Foxp1 was also expressed in the mantle zone. Nevertheless, it is still possible that Foxp1 is regulated by miR-150 as Foxp1 knockout mice shared a comparable pro-B to pre-B transition blockade phenotype with mice ectopically expressing miR-150 in hematopoietic cells. ${ }^{15,34}$ According to our Western blotting results, induction of miR-150 resulted in downregulation of c-Myb, Foxp1 and Survivin, supporting the targeting of these genes by miR-150. Nonetheless, we cannot exclude the possibility of an indirect regulatory pathway for the downregulation of Survivin and Foxp1.

It has been reported that c-Myc, which is implicated in the vast majority of $\mathrm{BL}$, can induce the expression of the miR-1792 cluster and repress the expression of miR-150. ${ }^{48,49}$ In line with the earlier publications, ${ }^{14,15}$ we observed a trend of increased percentage of apoptotic/death cells upon induction of miR-150 in the BL-derived cell line, DG75. Nevertheless, the increased percentage of apoptotic/death cells was not statistically significant. It can be speculated that the induction of 
miR-150 was not sufficient to suppress the c-Myc-mediated lymphomagenesis, as the expression levels of the oncogenic miR-17-92 cluster members remains high.

In conclusion, we have determined a miRNA profile of naïve, GC and memory B cells sorted from normal tonsils. miRNAs that were differentially expressed have been identified and verified, for the first time, by miRNA ISH in tonsil tissue sections. Remarkably, miRNA ISH revealed variation in the expression levels within the GC structures, which indicates the involvement of differentially expressed miRNAs in the functional regulation of centroblasts and centrocytes. Our findings warrant future studies in centroblasts and centrocytes aiming at elucidating the exact pathways targeted by these and possibly by other miRNAs.

Supplementary Information accompanies the paper on the Laboratory Investigation website (http://www.laboratoryinvestigation.org)

\section{ACKNOWLEDGEMENT}

This work is supported by the grant from the Ubbo Emmius Foundation, University Medical Center Groningen and from the Dutch Cancer Society, project number 2006-3643.

1. Lee RC, Feinbaum RL, Ambros V. The C. elegans heterochronic gene lin-4 encodes small RNAs with antisense complementarity to lin-14. Cell 1993;75:843-854.

2. Reinhart BJ, Slack FJ, Basson $M$, et al. The 21-nucleotide let-7 RNA regulates developmental timing in Caenorhabditis elegans. Nature 2000;403:901-906.

3. Bartel DP. MicroRNAs: genomics, biogenesis, mechanism, and function. Cell 2004;116:281-297.

4. Liang $\mathrm{Y}$, Ridzon $\mathrm{D}$, Wong $\mathrm{L}$, et al. Characterization of microRNA expression profiles in normal human tissues. BMC Genomics 2007;8:166.

5. Brennecke J, Hipfner DR, Stark A, et al. Bantam encodes a developmentally regulated microRNA that controls cell proliferation and regulates the proapoptotic gene hid in Drosophila. Cell 2003;113:25-36.

6. Xu P, Vernooy SY, Guo M, et al. The Drosophila microRNA Mir-14 suppresses cell death and is required for normal fat metabolism. Curr Biol 2003;13:790-795.

7. Wienholds $E$, Kloosterman WP, Miska E, et al. MicroRNA expression in zebrafish embryonic development. Science 2005;309:310-311.

8. Zhan M, Miller CP, Papayannopoulou T, et al. MicroRNA expression dynamics during murine and human erythroid differentiation. Exp Hematol 2007;35:1015-1025.

9. Fazi $F$, Rosa A, Fatica A, et al. A minicircuitry comprised of microRNA223 and transcription factors NFI-A and C/EBPalpha regulates human granulopoiesis. Cell 2005;123:819-831.

10. Ramkissoon SH, Mainwaring LA, Ogasawara Y, et al. Hematopoieticspecific microRNA expression in human cells. Leuk Res 2006;30: 643-647.

11. Georgantas III RW, Hildreth R, Morisot S, et al. CD34+ hematopoietic stem-progenitor cell microRNA expression and function: a circuit diagram of differentiation control. Proc Natl Acad Sci USA 2007;104:2750-2755.

12. Wu $\mathrm{H}$, Neilson $J R$, Kumar $P$, et al. miRNA profiling of naive, effector and memory CD8 T cells. PLoS ONE 2007;2:e1020.

13. Chen CZ, Li L, Lodish HF, et al. MicroRNAs modulate hematopoietic lineage differentiation. Science 2004;303:83-86.

14. Xiao C, Calado DP, Galler G, et al. MiR-150 controls B cell differentiation by targeting the transcription factor c-Myb. Cell 2007;131:146-159.

15. Zhou B, Wang S, Mayr C, et al. miR-150, a microRNA expressed in mature $B$ and $T$ cells, blocks early $B$ cell development when expressed prematurely. Proc Natl Acad Sci USA 2007;104:7080-7085.
16. Teng $G$, Hakimpour $P$, Landgraf $P$, et al. MicroRNA-155 is a negative regulator of activation-induced cytidine deaminase. Immunity 2008;28:621-629.

17. Vigorito $\mathrm{E}$, Perks $\mathrm{KL}$, Abreu-Goodger $\mathrm{C}$, et al. microRNA-155 regulates the generation of immunoglobulin class-switched plasma cells. Immunity 2007;27:847-859.

18. Li QJ, Chau J, Ebert PJ, et al. miR-181a is an intrinsic modulator of T cell sensitivity and selection. Cell 2007;129:147-161.

19. Fulci V, Chiaretti S, Goldoni M, et al. Quantitative technologies establish a novel microRNA profile of chronic lymphocytic leukemia. Blood 2007;109:4944-4951.

20. Wang M, Tan LP, Dijkstra MK, et al. miRNA analysis in B-cell chronic lymphocytic leukaemia: proliferation centres characterized by low miR-150 and high BIC/miR-155 expression. J Pathol 2008;215:13-20.

21. He L, Thomson JM, Hemann MT, et al. A microRNA polycistron as a potential human oncogene. Nature 2005;435:828-833.

22. Kluiver J, Poppema S, de Jong D, et al. BIC and miR-155 are highly expressed in Hodgkin, primary mediastinal and diffuse large B cell lymphomas. J Pathol 2005;207:243-249.

23. Eis PS, Tam W, Sun $L$, et al. Accumulation of miR-155 and BIC RNA in human B cell lymphomas. Proc Natl Acad Sci USA 2005;102: 3627-3632.

24. Costinean S, Zanesi N, Pekarsky $\mathrm{Y}$, et al. Pre-B cell proliferation and lymphoblastic leukemia/high-grade lymphoma in $E(m u)-m i R 155$ transgenic mice. Proc Natl Acad Sci USA 2006;103:7024-7029.

25. Baltimore $\mathrm{D}$, Boldin MP, O'Connell RM, et al. MicroRNAs: new regulators of immune cell development and function. Nat Immunol 2008;9:839-845.

26. Malumbres R, Sarosiek KA, Cubedo $E$, et al. Differentiation-stagespecific expression of microRNAs in B-lymphocytes and diffuse large B-cell lymphomas. Blood 2008; e-pub ahead of print.

27. Hogerkorp CM, Borrebaeck CA. The human CD77- B cell population represents a heterogeneous subset of cells comprising centroblasts, centrocytes, and plasmablasts, prompting phenotypical revision. J Immunol 2006;177:4341-4349.

28. Klein U, Tu Y, Stolovitzky GA, et al. Transcriptional analysis of the B cell germinal center reaction. Proc Natl Acad Sci USA 2003;100: 2639-2644.

29. Tjin EP, Bende RJ, Derksen PW, et al. Follicular dendritic cells catalyze hepatocyte growth factor (HGF) activation in the germinal center microenvironment by secreting the serine protease HGF activator. J Immunol 2005;175:2807-2813.

30. Sturn A, Quackenbush J, Trajanoski Z. Genesis: cluster analysis of microarray data. Bioinformatics 2002;18:207-208.

31. van den Berg A, Kroesen BJ, Kooistra K, et al. High expression of B-cell receptor inducible gene $\mathrm{BIC}$ in all subtypes of Hodgkin lymphoma. Genes Chromosomes Cancer 2003;37:20-28.

32. Lagos-Quintana $M$, Rauhut $R$, Yalcin $A$, et al. Identification of tissue-specific microRNAs from mouse. Curr Biol 2002;12:735-739.

33. Kluiver J, Haralambieva $\mathrm{E}$, de Jong $\mathrm{D}$, et al. Lack of BIC and microRNA miR-155 expression in primary cases of Burkitt lymphoma. Genes Chromosomes Cancer 2006;45:147-153.

34. Hu $\mathrm{H}$, Wang $B$, Borde $M$, et al. Foxp1 is an essential transcriptional regulator of B cell development. Nat Immunol 2006;7:819-826.

35. Thomas MD, Kremer CS, Ravichandran KS, et al. c-Myb is critical for B cell development and maintenance of follicular B cells. Immunity 2005;23:275-286.

36. Liu YJ, Malisan F, de Bouteiller O, et al. Within germinal centers, isotype switching of immunoglobulin genes occurs after the onset of somatic mutation. Immunity 1996;4:241-250.

37. Pascual V, Liu YJ, Magalski A, et al. Analysis of somatic mutation in five B cell subsets of human tonsil. J Exp Med 1994;180:329-339.

38. Allen CD, Okada T, Cyster JG. Germinal-center organization and cellular dynamics. Immunity 2007;27:190-202.

39. Ivanovska I, Ball AS, Diaz RL, et al. MicroRNAs in the miR-106b family regulate $\mathrm{p} 21 / \mathrm{CDKN} 1 \mathrm{~A}$ and promote cell cycle progression. Mol Cell Biol 2008;28:2167-2174.

40. Taganov KD, Boldin MP, Chang KJ, et al. NF-kappaB-dependent induction of microRNA miR-146, an inhibitor targeted to signaling proteins of innate immune responses. Proc Natl Acad Sci USA 2006;103:12481-12486.

41. Sen R. Control of B lymphocyte apoptosis by the transcription factor NF-kappaB. Immunity 2006;25:871-883. 
42. de Yebenes VG, Belver L, Pisano DG, et al. miR-181b negatively regulates activation-induced cytidine deaminase in B cells. J Exp Med 2008;205:2199-2206.

43. Hamblin TJ, Davis Z, Gardiner A, et al. Unmutated Ig V(H) genes are associated with a more aggressive form of chronic lymphocytic leukemia. Blood 1999;94:1848-1854.

44. Klein U, Tu Y, Stolovitzky GA, et al. Gene expression profiling of B cell chronic lymphocytic leukemia reveals a homogeneous phenotype related to memory B cells. J Exp Med 2001;194:1625-1638.

45. Gewirtz AM, Calabretta B. A c-myb antisense oligodeoxynucleotide inhibits normal human hematopoiesis in vitro. Science 1988;242: 1303-1306.
46. Nakata Y, Shetzline S, Sakashita C, et al. c-Myb contributes to G2/M cell cycle transition in human hematopoietic cells by direct regulation of cyclin B1 expression. Mol Cell Biol 2007;27: 2048-2058.

47. Anfossi G, Gewirtz AM, Calabretta B. An oligomer complementary to c-myb-encoded mRNA inhibits proliferation of human myeloid leukemia cell lines. Proc Natl Acad Sci USA 1989;86: 3379-3383.

48. Chang TC, Yu D, Lee YS, et al. Widespread microRNA repression by Myc contributes to tumorigenesis. Nat Genet 2008;40:43-50.

49. O'Donnell KA, Wentzel EA, Zeller KI, et al. c-Myc-regulated microRNAs modulate E2F1 expression. Nature 2005;435:839-843. 